Cad.Est.Ling., Campinas, (44):57-70, Jan./Jun. 2003

\title{
LA CONSTRUCTION DE LA RÉFÉRENCE COMME TRAVAIL INTERACTIF: ACCOMPLIR LA VISIBILITÉ DU DÉTAIL ANATOMIQUE DURANT UNE OPÉRATION CHIRURGICALE
}

\author{
LORENZA MONDADA \\ (Université Lyon II)
}

\begin{abstract}
RESUMO Tanto no campo da Filosofia da Linguagem, quando no da Lingüística, a questão da referência recebe tratamento diversificado: para uns, a referência é concebida a partir de um modelo de correspondência entre as palavras do discurso e os objetos do mundo; para outros, a referência é o resultado de um processo dinâmico e sobretudo intersubjetivo. Enquanto que a primeira posição encontra abrigo em uma longa tradição lógico-filosófica, a segunda se caracteriza por reflexões advindas das ciências humanas e sociais, empiricamente sustentadas e discutidas. Tomada a referência como prática, vista a partir da atividade e não a partir do sistema da língua, o deslocamento teórico da questão é capaz de, entre outras coisas, colocar em xeque a distinção entre o que é intra e extra-discursivo.
\end{abstract}

\section{INTRODUCTION: DES APPROCHES PLURIELLES DES PRATIQUES RÉFÉRENTIELLES}

1.1. La question de la référence traverse la philosophie du langage et la linguistique, en prenant des formes théoriques différenciées: pour les uns, la référence est conçue dans un modèle de la correspondance entre les mots du discours et les objets du monde, les seconds évaluant la vérité des premiers dans un cadre vériconditionnel; pour les autres, la référence est le résultat d'un processus dynamique et avant tout intersubjectif, qui s'établit dans le cadre des interactions entre locuteurs et qui est susceptible de se transformer au fil des déroulements discursifs, des accords et des désaccords. Alors que la première position tire ses lettres de noblesse d'une longue tradition philosophique et logique (cf. Nelson, 1992), la seconde relève davantage de réflexions issues des sciences humaines et sociales, qui ont bénéficié, outre des apports de courants théoriques (comme le pragmatisme, la phénoménologie, la théorie sociale, l'interactionnisme symbolique), de l'ouverture contemporaine de la linguistique aux données empiriques recueillies sur des terrains authentiques, différenciés, complexes, très éloignés des données de cabinet fournies pendant longtemps aux linguistes par leur introspection.

1.2. L'analyse des pratiques référentielles des locuteurs découle de ces choix épistémologiques, et ceci contribue à en expliquer la grande diversité dans la littérature 
contemporaine.Si la référence n'est plus généralement considérée comme un problème strictement linguistique, mais comme un phénomène concernant à la fois la cognition et les usages du langage en contexte et en société (Koch, 1989, 1993, 1997), il subsiste aujourd'hui une multiplicité de cadres théoriques différents disponibles pour l'appréhender. D'une part, les approches cognitives renvoient la gestion des activités de référenciation à des savoirs partagés dont dépendent les choix des expressions référentielles adéquates, i.e.à des états cognitifs caractérisant non seulement le locuteur mais aussi son interlocuteur et la manière dont il est appréhendé par le premier (Givon, 1992; Gundel et alii, 1993; Lambrecht, 1994). Les approches cognitives peuvent donc se centrer soit sur le sujet, soit sur l'établissement d'une intersubjectivité -soit-elle traitée en termes de "mémoire discursive" ou de "cognition distribuée" (p.ex.Clark \& Wilkes-Gibbs, 1986; Clark \& Brennan, 1991). D'autre part, l'alternative aux approches cognitives est constituée par les approches interactionnistes, résolumment centrées sur ce qui est publiquement déployé par les participants -ce qui est rendu pertinent, reconnaissable, intelligible par eux (accountable ) - plutôt que sur des processus mentaux internes aux sujets. Dans cette perspective, ce sont les pratiques référentielles manifestées dans l'interaction sociale qui font l'objet de l'analyse -pratiques langagières mais aussi pratiques gestuelles, mouvements dans l'espace, orientation des regards; les "référents" visés par ces pratiques ne sont pas traités comme leur préexistant en tant que tels, mais comme instaurés dans l'accomplissement et le déroulement de l'activité référentielle, par la manière même dont celle-ci est reconnaissablement organisée (Geluykens, 1995; Ford \& Fox, 1996; Pekarek, 1998; Mondada, 1994, 2002, à paraître b).

1.3. L'analyse des ressources formelles mobilisées dans les activités référentielles dépend largement des options esquissées ci-dessus: les choix formels peuvent être conçus comme étant des reflets de propriétés du référent, ou bien comme manifestation d'états mentaux ou bien encore comme l'exploitation de ressources dans l'établissement d'un accord intersubjectif ou d'un alignement rendant pertinent, visible et présent un référent qui est traité comme un objet de discours avant que comme un objet du monde. Dans ce dernier cas, on ne parlera pas tellement de marqueurs d'un statut référentiel que de ressources langagières (Mondada, 2001a) mobilisées pour accomplir des propriétés référentielles (comme la plus ou moins grande disponibilité du référent à l'attention des participants ou comme l'identification, le pointage et la délimitation de détails pertinents à l'activité en cours). Nous allons montrer que cet accomplissement est réalisé par le langage mais aussi par le geste et l'usage situé d'artéfacts -ces trois types de ressources étant exploités de manière finement synchronisée et intégrée.

1.4. C'est dans ce cadre que nous allons nous concentrer sur une ressource formelle particulière, l'invocation de la vision au moyen de l'expression "you see", utilisée avec ou sans $\mathrm{SN}$, que nous allons traiter comme un procédé pour accomplir la visibilité des objets pointés ou mentionnés. Nous allons le faire sur des données extraites d'un corpus relativement 'exotique'pour les linguistes: des interactions entre chirurgiens durant leur travail en salle d'opération (cf. Mondada, 2001b, à paraitre c). 
Cette problématique nous a été inspirée par la spécificité des données considérées, apparaissant comme un trait particulier et récurrent du corpus; en même temps il s'agit de phénomènes qui caractérisent d'autres interactions professionnelles fondées sur un exercice de la "vision professionnelle", faisant intervenir un regard compétent et des descriptions compétentes de ce que définit ce regard. De manière générale, les phénomènes étudiés ici permettent une réflexion sur la référence à des propriétés visuelles dans le discours et dans la langue en interaction.

1.5. Les usages de "you see"dans ce corpus permettent de revisiter une problématique récurrente dans la littérature sur la référence ou sur la topicalité: la place des propriétés de visibilité du référent dans le choix des stratégies référentielles.

En effet, on trouve dans la littérature sur le topic et sur la deixis des renvois intéressants à la visibilité des objets.Ainsi, par exemple, la propriété de visibilité du topic est présente dans la définition du topic chez van Oosten: "What a person speaks about is the focus of the speaker's attention and of the hearer's attention and thus in their consciousness; it is something that the speaker is interested in and that is the perspective from which the speaker is viewing the event ; it is present in the immediate environment, concrete and visible; and it is salient in the speaker's perception of the event." ((1984, 46). D'autre part, différents travaux sur la deixis spatiale ont fait observer que les systèmes de certains démonstratifs faisaient intervenir comme critère oppositif, outre la distance de l'entité repérée du locuteur et/ou de l'auditeur, la visibilité de cette entité (par exemple le Quileute, qui a 3 démonstratifs pour les objets invisibles et 4 pour les visibles), ainsi que son marquage comme information ancienne ou nouvelle (par exemple le Hausa qui distingue entre entité nouvelle proche/distante, entité mentionnée proche/distante) (Anderson \& Keenan, 1985). Langacker analysant l'opposition $u / a$ en Cora, montre qu'elle fait intervenir des critères tels que l'intériorité/extériorité, la présence dans/hors le champ visuel, ainsi que l'accessibilité/inaccessibilité de l'objet $(1990,52)$. Un rapport très étroit semble donc exister, au sein du système linguistique lui-même entre deixis et topicalité, entre visibilité et mentionnabilité des entités.

Par ailleurs, "you see"a été traité dans la littérature comme un discourse marker (Schiffrin, 1987) en voie de grammaticalisation, proche de "you know", renvoyant de manière métaphorique à l'établissement d'un espace intersubjectif commun aux interlocuteurs, et plus particulièrement comme opérant des transitions interactionnellement co-construites dans la connaissance partagée et par là d 'éventuels alignements entre les interlocuteurs.

Ce marqueur a aussi été traité comme un procédé d'introduction de topics "brand new" (Lambrecht, 1986, 1990), dans des exemples comme les suivants:

Tout d'un coup j'entends broum broum . j'dis qu'est-ce qui s'passe . hein . on était à deux . on sort d'un seul coup . qu'est-ce qu'on voit . une voiture qu'avait . par-dessus le parapet . qu'était plongée dans la Seine (Lambrecht, 1986, 289, 7.52)

J'vois les jeunes qui disent ah vivement les vacances (idem, 291, 7.55) 
Les exemples donnés par Lambrecht privilégient des constructions du type Vvoir +le/un $N$ +relative, qu'il rapproche des avoir-cleft constructions ("Y avait une voiture qui était dans la Seine") et qui constituent des techniques d 'introduction d 'un nouvel objet dans le discours. Du point de vue pragmatique, Lambrecht se limite à observer que dans certains cas le verbe voir est utilisé littéralement et dans d'autres métaphoriquement: "The use of the verb voir in the perception verb construction may be seen as a semantic transfer whereby the perception of a referent is metaphorically interpreted as its presentation in the discourse" $(1986,291)$. De cette manière, l'invocation de la vision serait une macro-procédure pour obtenir l'attention de l'interlocuteur, pour rendre accessible et identifiable le topic, pour le rendre reconnaissable en tant que tel, et souvent, pour en motiver, justifier, légitimer l'introduction. Ce procédé rend ainsi palpables un certain nombre de contraintes généralement reconnues comme pesant sur toute gestion de la référence.

Or, contrairement à cette littérature, où "you see"comme marqueur tend à être fortement désémantisé au fur et à mesure de sa grammaticalisation, le corpus enregistré en chirurgie sur lequel nous avons travaillé met en oeuvre une "vision professionnelle" (Goodwin, 1994) qui s'exerce sur des détails effectivement vus et rendus visibles par leur description et les gestes qui l'accompagnent.

1.6. Face aux données sur lesquelles s'est fondée la littérature existante, il peut donc être intéressant de se tourner vers des situations où la vision et la description de la vision n'a pas un sens métaphorique et où son interprétation ne relève pas uniquement d'hypothèses que ce sens métaphorique permet d'échafauder sur le fonctionnement cognitif ou représentationnel des processus référentiels. Ces situations ne sont pas marginales dans la culture et dans la société dans lesquelles nous vivons: au contraire, elles concernent des domaines très divers de la vie professionnelle, où le travail sur les visualisations joue un rôle central. Tel est le cas par exemple des professionels utilisant des caméras de surveillance (Ball, 2000), des stations de cntrôle dans les aéroports (Goodwin \& Goodwin, 1996; Suchman, 1996), ou dans les métros (Luff \& Heath, 2000; Luff, Heath \& Jirotka, 2000), ou encore dans des situations de travail fortement organisées par les médiations technologiques (Luff \& Heath, 1993). On peut dire que ces espaces complexes fournissent des terrains empiriques sur lesquels interroger les pratiques référentielles liées à la vision, y compris les pratiques de production d'effets de référenciation, d'objectivation, de facticité (Lynch, 1988), comme les pratiques de raisonnement, de reconnaissance perceptive, de lecture visuelle (Hartswood, Procter, Rouncefield, \& Slack, 2000).

Ces pratiques obligent la linguistique à ne pas se limiter à rendre compte d'activités des locuteurs qui seraient exclusivement verbales et à reléguer les autres processus dans le domaine de la cognition. Elles obligent au contraire à prendre en considération des situations -nombreuses -où la parole est imbriquée dans l'action nonverbale, dans la matérialité du contexte et dans le maniement d'objets. Ceci nous paraît fondamental pour une réflexion sur la production de la référence -qui se fait à travers des pratiques sociales multi-modales et non seulement linguistiques (Mondada, à paraître a). 
Ces pratiques sont interactives: c'est en observant le déroulement séquentiel de l'interaction que l'intelligibilité et le sens des processus intersubjectifs sont rendus manifestes -pour les participants avant même que pour les analystes. En enchaînant d'une certaine façon par rapport à ce qui précède, en rendant manifeste une absence, en traitant un élément précédemment introduit d'une certaine manière, les locuteurs rendent publiquement manifestes leurs processus interprétatifs aux fins pratiques de l'activité en cours. Si l'on s'intéresse aux processus de référenciation, comme à d'autres types de processus linguistiques et discursifs, les données interactionnelles authentiques, enregistrées dans des contextes non provoqués et non contrôlés par le chercheur, ne constituent pas uniquement un terrain fécond où mettre à l'épreuve des théories élaborées par ailleurs mais bien des pratiques sociales dont l'observation peut amener à réviser certains présupposés théoriques.

\section{2. “YOU SEE" AU DÉBUT DU TOUR: LA DÉMONSTRATION ANATOMIQUE}

Le phénomène particulier qui nous intéresse ici concerne un certain nombre d'occurrences du verbe "voir"qui fonctionnent comme une ressource dans l'introduction et le traitement référentiel des objets de discours qu'elles permettent de mettre en avant. Tel est le cas du fragment ci-dessous:
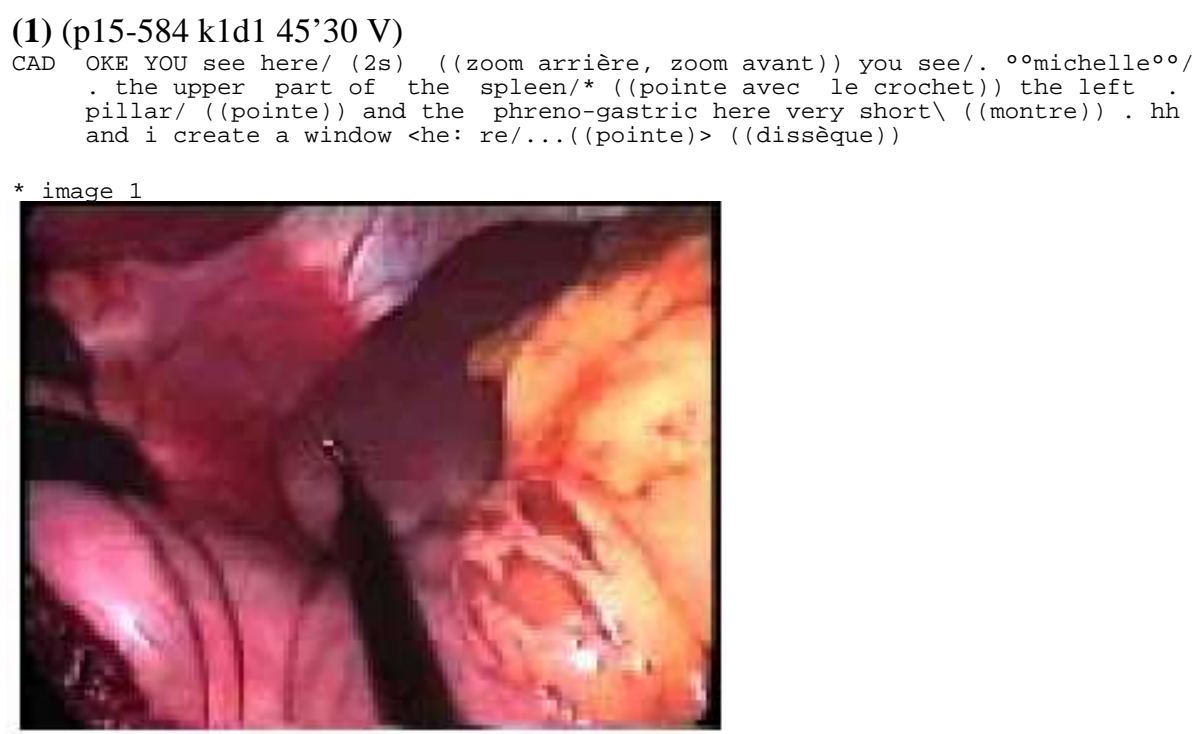

Cette description a lieu au sein d'une activité récurrente dans les opérations chirurgicales que nous avons enregistrées, où le chirurgien en chef explique la procédure à des chirurgiens moins expérimentés, qui la regardent à distance, grâce à sa transmission sur des moniteurs vidéo. Le type de procédure observée ici se prête bien à 
l'exercice, puisqu'il s'agit de chirurgie laparoscopique, pratiquée en insérant dans le corps du patient une caméra endoscopique qui retransmet l'image anatomique sur un moniteur dans la salle d'opération.

L'extrait cité relève de la "démonstration anatomique", un voyage à l'intérieur de l'espace du corps au cours duquel des détails anatomiques sont rendus pertinents, voire visibles, par le chirurgien.La description est introduite par le verbe "voir"à la deuxième personne ("you see"), accompagné de déictiques spatiaux ("here"), et localisant des objets, introduits par les syntagmes nominaux définis, souvent énumérés dans une liste. En outre, l'enregistrement vidéo de ces descriptions nous montre qu'elles sont accompagnées par des gestes importants, réalisés avec les instruments chirurgicaux qui cessent d'être utilisés pour disséquer, écarter, tendre des tissus et sont exploités comme des pointeurs. Même si dans ce fragment aucun autre locuteur n'intervient, la description est adressée à plusieurs destinataires, manifestés par la deuxième personne et par le prénom ("michelle"). Cette dernière est l'assistante du chirurgien, qui tient la caméra endoscopique et qui est donc responsable des mouvements du zoom: même si c'est le chirurgien qui énonce la description, l'activité référentielle à laquelle il se livre est une activité distribuée, accomplie collaborativement par sa parole, ses instruments et par la caméra manoeuvrée par Michelle. En outre, le chirurgien s'adresse par la deuxième personne à l'audience des médecins en formation continue pour qui l'activité de monstration de l'anatomie est organisée et transmise, en tenant notamment compte de leurs compétences, matérialisées dans le type de "vision professionnelle" dont ils font preuve, et dont l'exercice produit le caractère évident ou au contraire illisible de l'anatomie.

Ainsi, la description de l'anatomie ne se limite pas à verbaliser des référents qui seraient déjà là, disponibles et visibles; tout au contraire, par la manière dont elle est organisée, par les syntagmes nominaux et les gestes de pointage qui la constituent, la description rend visibles voire constitue les référents, en sélectionnant certains détails pertinents à l'exclusion d'autres détails passés sous silence, en organisant un réseau de repères anatomiques adéquats pour le type d'opération en cours. En même temps, l'usage du défini traite ces choix comme allant de soi, comme s'imposant au discours, au regard et à l'action. De même, la vision qui est sollicitée par "you see" n'est pas une simple perception du monde déjà là, mais une pratique sociale spécialisée qui intervient activement dans l'espace parfois indistinct du corps, en s'associant au maniement du scalpel qui a précisément la fonction de rendre distincts et reconnaissables les tissus et les organes sur lesquels intervient ensuite le chirurgien. Cet enchaînement entre démonstration anatomique et action chirurgicale est d'ailleurs visible dans le fait que la description aboutit à la dissection.

Cette activité descriptive est récurrente, ainsi que le montrent d'autres occurrences du même type:

(2) DAC euh: <you see here. the extragastric nerve/ ((montre avec la pince))
$(($ zoom $))>$. okay/. sm- small nodes ((montre)) <. this is the laxxxxx nerve/ ( (montre) $)>$ and <here .. the (crow-foot) \( (montre)) > okay/ 
MIL and here you see a beautiful view/ of the epigastric vessels $\backslash$ (1) the epigastrics to the right $\backslash$ there's the double vein here and you see (1) a big - epigastric artery $\backslash$. here is/. the area where the hernia will be probably

Ces explorations anatomiques peuvent prendre une forme plus ou moins didactique, exhiber le fait que le chirurgien traite son auditoire comme (re) connaissant plus ou moins la région anatomique indiquée -mais en amont de ces variations elles se déroulent en suivant une séquence fortement typée.

\section{3. "YOU SEE" À LA FIN DU TOUR: LA RECHERCHE D'UNE CONFIRMATION À PROPOS D'UNE LOCALISATION}

Face à ces occurrences, un autre type de description, à la fois proche par le type de ressources linguistiques mobilisées et différent par leur organisation séquentielle, est observable, comme dans les extraits suivants:

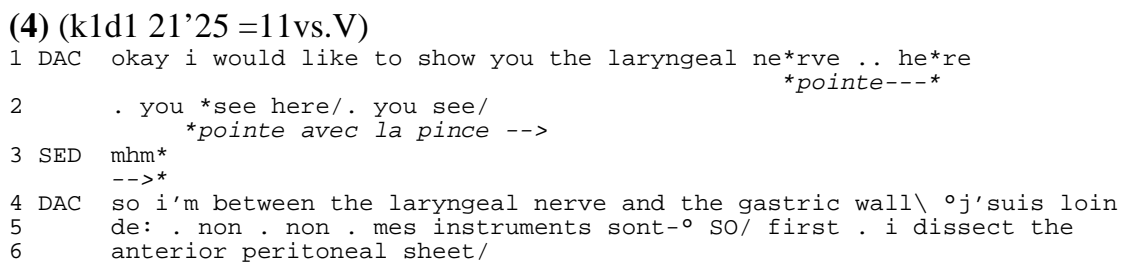

Nous retrouvons ici les mêmes ressources linguistiques, le verbe "voir"à la deuxième personne, les déictiques, les syntagmes définis. Toutefois nous avons affaire à un autre type de séquence et à un autre type de construction interactive de la référence, qui relève d'une distribution séquentielle différente de ces ressources. En effet, ces deux extraits se caractérisent par le fait que le verbe "voir"est placé après la mention des repères anatomiques (sous forme définie) et plus précisément à la fin du tour de parole, dans une prosodie interrogative - alors que dans les premiers cas le verbe était placé au début du tour de parole, à la forme affirmative. "You see" traite ici l'interlocuteur de manière différente, en s'adressant à lui de façon à attendre de lui une réponse, une confirmation. Cette attente est visible dans le fait que son absence est observablement traitée par l'énonciateur, qui après une première occurrence de " (you) see here/" (extr.4: 2, extr.5: 2) ménage pour son interlocuteur une opportunité de prendre la parole. Comme celui-ci ne saisit pas l'occasion qui lui est offerte, le chirurgien relance son interpellation (extr.4: 2 "you see/", extr.5: 2: "you see this line 
here/") jusqu'à ce qu'elle reçoive une réponse de l'interlocuteur. Cette réponse construit le partage intersubjectif de la référence, qui n'est pas du tout considéré comme allant de soi par les partenaires, mais au contraire comme devant être activement assuré par une mise en forme adéquate du tour de parole. Ce n'est qu'ensuite que le chirurgien procède à la poursuite de la procédure, consistant à disséquer la région anatomique qui vient d'être explorée et reconnue.

L'importance de cet accord autour de la référence est rendu visible par l'insistance du chirurgien dans le cas suivant:

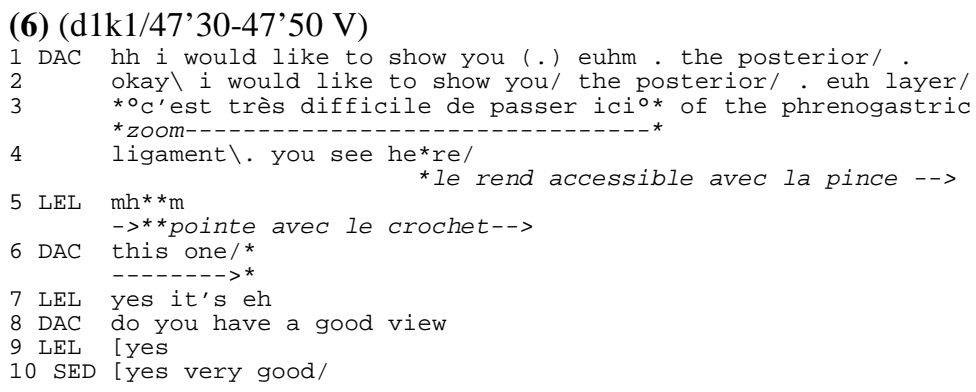

De manière intéressante, on a ici d'abord une annonce (par "i would like to show you"1) qui sélectionne une description définie; cette annonce est abandonnée puis reénoncée (dans un restart), et par là amplifiée progressivement. Ce déploiement progressif de l'annonce accompagne un geste difficile, formulé comme tel (3) dans une alternance entre l'anglais et le français, traitant la formulation comme destinée à l'équipe chirurgicale (française) avant qu'à l'audience (internationale). Ce geste, accompagné d'un zoom de la caméra, est doté de sa propre temporalité, qui investit celle de l'énonciation. Il contribue à l'accomplissement de la disponibilité et de la visibilité du référent. Celles-ci sont accentuées par le geste suivant, par lequel la pince dispose et déploie l'espace qui est donné à voir, en même temps qu'est énoncé le déictique ("you see here/"4). Pendant que le collègue expert répond (5), le chirurgien précise le geste, en pointant avec son crochet sur le détail visé; même si le Dr. Lelacq répond, le chirurgien précise encore sa visée référentielle par un démonstratif, toujours en pointant avec son crochet (6). Cette fois la réponse est plus décidée (7) mais elle est encore relancée par une question sur la qualité de l'image, qui reçoit cette fois non une mais deux réponses (11-12).

Cette séquence exhibe ainsi l'orientation du chirurgien vers ses destinataires et la manière dont les procédés référentiels utilisés sont progressivement renforcés par lui: dans le passage du syntagme défini au déictique, au démonstratif et à l'évaluation de l'image. 


\section{UNE RÉFÉRENCE VISUELLE ÉTABLIE COLLECTIVEMENT ET PAR UN TRAVAIL DE MONSTRATION}

Afin d'approfondir la dimension interactionnelle de ces procédés d'élaboration de la référence, ainsi que la dimension collaborative du regard et de la vision, on se penchera sur un dernier extrait, reproduit d'abord dans une transcription simplifiée et que nous reprendrons ensuite dans une transcription plus élaborée:

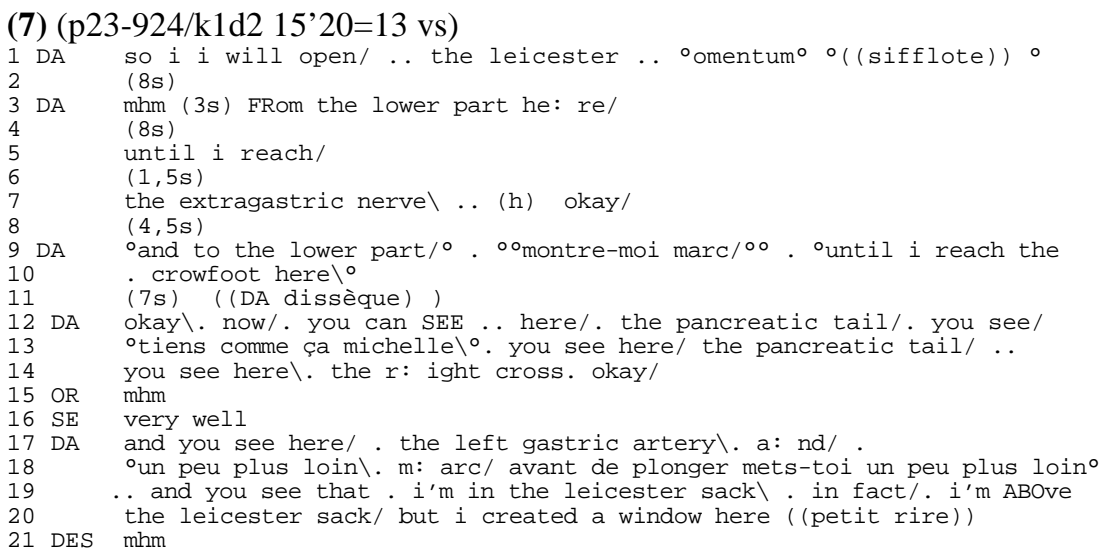

L'invitation à regarder l'anatomie -on retrouve ici aux lignes 12-14, puis 17-20 des fragments de description analogues à ceux que nous venons d'analyser dans le chapitre 2 -va de pair avec une activité consistant à la montrer, les deux étant étroitement articulées. Montrer, pointer, indiquer, centrer des détails anatomiques sont des actions accomplies par le chirurgien de manière collaborative, comme nous l'esquissions dès le premier extrait.Si le chirurgien en chef énonce la parole explicative, si c'est lui qui tient le crochet et qui dissèque, ses assistants sont des aides indispensables, l'aidant à structurer le champ opératoire avec des pinces, ou manoeuvrant la caméra endoscopique. En particulier, nous allons montrer comment l'activité de la description est finement coordonnée avec celle de la manipulation de la caméra, comportant des déplacements mais aussi des zooms en avant et en arrière. C'est pourquoi il est important d'intégrer dans la transcription et dans l'analyse la prise en compte non seulement des détails du déroulement de la parole-en-interaction, mais aussi de ceux des gestes et des mouvements de la caméra, que nous avons transcrit en utilisant des conventions spécifiques et sur des lignes distinctes dans une nouvelle version de l'extrait (cf. Mondada, à paraître d). L'opération vise à traiter l'obésité chronique et consiste à entourer une partie de l'estomac d'un anneau qui en diminue ainsi le volume -et donc l'appétit du patient: 


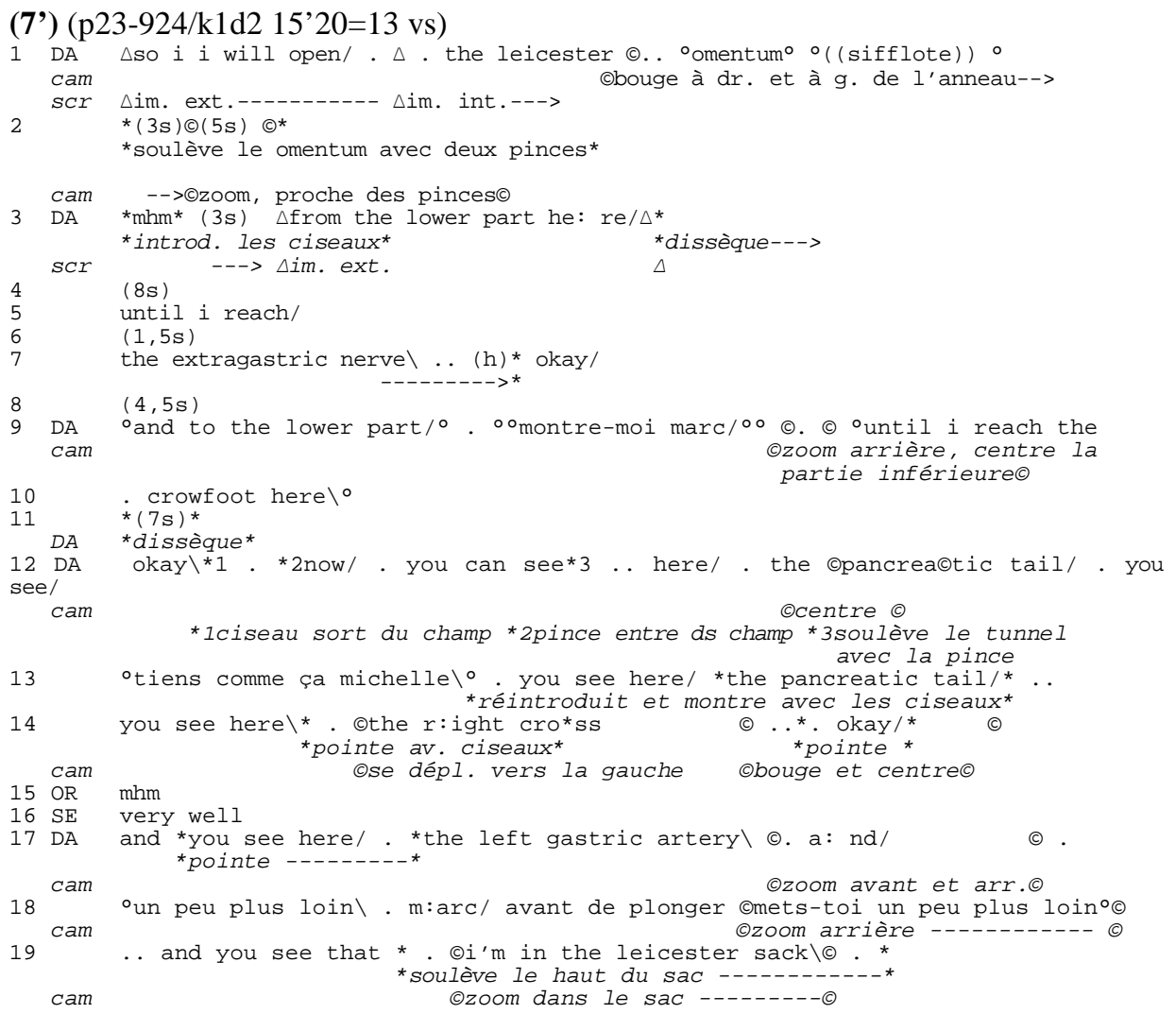

Le Dr. Daccard procède successivement à la dissection et à la démonstration de l'anatomie. Les deux demandent des mouvements spécifiques de la caméra vidéo, tenue par l'assistant, Marc; ces mouvements sont localement structurés au fil de l'interaction entre les deux professionnels, en s'ajustant de manière spécifique aux différentes tâches.

La séquence commence par "I will open" (1), une description au futur qui projette l'action successive, décrite au fur et à mesure qu'elle se déploie, dans un seul énoncé complexe (1-10). L'organisation séquentielle de cet énoncé est configurée non par la syntaxe mais par l'action chirurgicale à laquelle elle est synchronisée: le rythme de la mention des différents repères anatomiques dépend de la progression de la dissection au ciseau, qui progressivement atteint et dépasse ces localisations.

Le travail de la caméra suit l'action des ciseaux: au début (1) il rencontre quelques problèmes à cause de l'anneau qui entrave le champ de la vision et qui, par sa blancheur, absorbe la plupart de la lumière; puis (2) il zoome sur l'omentum où il se fixe, avant de bouger vers la partie inférieure du champ (9). Contrairement aux autres, ce dernier mouvement est explicitement requis par Daccard à son assistant ("montremoi marc"9). Une fois que la caméra est recentrée sur le nouveau repère elle se stabilise 
à nouveau. Les mouvements de la caméra sont donc minimisés, celle-ci restant le plus fixe possible une fois que la bonne prise de vue a été trouvée.

A la fin de la dissection, "okay" (12) rend manifeste la transition à autre activité, la démonstration anatomique: l'instrument qui servait à disséquer disparaît et les pinces sont introduites, qui permettent de rendre l'espace disponible et visible.Les mouvements des instruments sont effectués de sorte à rendre la description intelligible, notamment pour exhiber les repères indiqués par le déictique "here" $(12,13,14)$. L'activité principale est ici le pointage pour le public, organisé à la fois par la parole et par le gestes effectués avec les pinces. L'organisation des tours est finement synchronisée avec les gestes de pointage. Daccard s'oriente vers cette coordination, comme le montre la réparation de la première version de la description (12) qui est reprise (13) après qu'une bonne exposition du champ ait été produite, au moyen des pinces. Une fois le champ préparé, celles-ci sont ensuite passées à Michelle, ce qui laisse les mains de Daccard libres pour reprendre les ciseaux, utilisés cette fois pour pointer et non pour disséquer. La deuxième version de la description est coordonnée avec des gestes de pointage absents de la première. Le travail de la caméra est maintenant organisé en s'ajustant à de nouvelles pertinences: elle se centre sur l'ouverture pratiquée par Daccard pour montrer la queue du pancréas (12), puis sur le repère suivant (14) et zoome enfin sur le successif (17). Le dernier mouvement est réparé par la requête de Daccard (18) qui initie une stratégie de monstration plus complexe -consistant en un zoom arrière pour donner une vue générale et puis un zoom avant qui pénètre dans le tunnel). Durant la phase de la démonstration, le travail de la caméra est plus mobile, et obéit moins aux impératifs de la bonne exposition du champ à disséquer qu'aux parcours descriptif, plus mobile, qui organise la démonstration anatomique. Donc les mouvements de la caméra sont très différents pour la dissection et pour la description: la première privilégie la stabilité, la seconde la mobilité, articulée avec le déroulement de la description verbale. "You see" préface l'accomplissement de la visibilité pour l'audience selon le second mode.

\section{EN GUISE DE CONCLUSION}

$\mathrm{Au}$ terme de ces observations, on peut dire que la description de l'anatomie est réalisée en mobilisant une série de ressources linguistiques (les expressions référentielles classiquement décrites dans la littérature -cf. Charolles, 2000) qui toutefois à elles seules n'épuisent pas le processus référentiel. Celui-ci a lieu au cours d'une activité qui est autant discursive que gestuelle; c'est au sein de son organisation temporelle et séquentielle que les ressources linguistiques sont placées et exploitées par les locuteurs. L'appel à la vue (manifestée notamment par "you see") devient dans ce contexte un dispositif complexe de production référentielle, où la parole, le geste, l'instrument chirurgical, la disposition de l'espace anatomique au moyen des pinces et de la dissection contribuent ensemble à l'accomplissement de la référence.

Ces observations analytiques invitent à un déplacement théorique de la problématique de la référence d'un cadre statique abstrait, où les forme linguistiques 
sont traitées pour elles- mêmes dans leur mise en correspondance ou non avec des référents extra-discursifs, à un cadre dynamique, centré sur des pratiques de référenciation qui impliquent une organisation non seulement de la parole mais aussi de l'espace et du contexte où elle s'énonce, et donc de la matérialité qui est modifiée en même temps que la parole est énoncée. La distinction entre ce qui est intra-et extradiscursif n'a plus lieu d'être dans ce cadre, qui est centré sur l'activité et non sur le système de la langue; cette activité intervient sur la référence en même temps qu'elle présente éventuellement celle-ci dans sa factualité et son objectivité. C'est dans ce sens qu'on peut parler de la référence comme d'un accomplissement pratique.

\section{CONVENTIONS DE TRANSCRIPTION}

\begin{tabular}{|c|c|c|c|}
\hline & chevauchements & . ..... & pauses \\
\hline$(2 s)$ & pauses en secondes & $\mathrm{xxx}$ & segment inaudible \\
\hline 11 & intonation montante/descendantel & exTRA & segment accentué \\
\hline$(($ rire $))$ & phénomènes décrits & & allongement vocalique \\
\hline$<>$ & délimitation des phénomènes entre (( )) & par- & troncation \\
\hline$\&$ & continuation du tour de parole & $=$ & enchaînement rapide \\
\hline$\wedge$ & liaison & (h) & aspiration \\
\hline (il va) & essai de transcription & ${ }^{\circ}$ bon ${ }^{\circ}$ & murmuré \\
\hline$* *$ & indication du début/de la fin d'un geste & & \\
\hline (C) & d'un mouvement de caméra & & \\
\hline$\Delta$ & ou d'un écran décrit à la ligne suivante & & \\
\hline $\mathrm{am}$ & mouvements de caméra & & \\
\hline & image apparaissant à l'écran (vue interne & endoscol & que $v s$ vue externe) \\
\hline$->$ & continuation du geste aux lignes suivante & & \\
\hline m3 & $\begin{array}{l}\text { situe exactement l'emplacement de l'ima } \\
\text { par rapport à la parole transcrite }\end{array}$ & ge tire & bande vide \\
\hline
\end{tabular}

\section{$\overline{\text { BIBLIOGRAPHIE }}$}

ANDERSON, S.R. \& E.L. Keenan (1985). "Deixis", in Shopen, T. (ed. ), Language Typology and Syntactic Description. III. Grammatical Categories and the Lexicon, Cambridge: Cambridge University Press, 259-308.

BALL, M. (2000). The visual availability and local organization of public surveillance systems: the promotion of social order in public space. Sociological Research Online (www. socresonline. org. $u k), 5(1)$.

CHAROLLES, M. (2002). La référence et les expressions référentielles en français. Paris: Ophrys.

CLARK, H. H. \& BRENNAN, S.E. (1991). Grounding in communication. In L.B. Resnick \&J. Levine \& S. D. Behreno (Eds. ), Perspectives on Socially Shared Cognition. Washington: American Psychological Association. 
CLARK, H.H. \& WILKES-GIBBS, D. (1986). Referring as a collaborative process. Cognition, 22, 1-39.

DIK, S.C. (1989). The Theory of Functional Grammar, Dordrecht: Fortis.

FORD, C.E. \& FOX, B.A. (1996). Interactional motivation for reference formulation: he had. This guy had, a beautiful, thirty-two O: lds. In B. Fox (Ed. ), Studies in Anaphora. Amsterdam: Benjamins.

GELUYKENS, R. (1995). On establishing reference in conversation. In R. A. Geiger (Ed. ), Reference in Multidisciplinary Perspective. Philosophical Object, Cognitive Subject, Intersubjective Process (pp. 230-240). Hildesheim: Georg Olms.

GIVON, T. (1992). The grammar of referential coherence as mental processing instructions. Linguistics, 30 (5-55).

GOODWIN, C. (1994). Professional Vision. American Anthropologist, 96 (3), 606-633.

GOODWIN, C. \& Goodwin, M.H. (1996). Seeing as a situated activity: Formulating planes. In D. Middleton $\&$ Y. Engestrom (Eds. ), Cognition and Communication at Work. Cambridge: Cambridge University Press.

GUNDEL, J.K.; HEDBERG, N. \& ZACHARSKI, R. (1993). Cognitive status and the form of referring expressions in discourse. Language, 69, 279-307.

HARTSWOOD, M.; PROCTER, R.; ROUNCEFIELD, M. \& SLACK, R. (2000). Interactional Practices in Breast Screening Work: A Case Study of Accountability and Professional Vision. Paper presented at the Paper given at the Medical Interaction Conference University of Southern Denmark, October 2000 .

KOCH, I.G.V. (1989). A coesão textual. Sao Paulo: Contexto. (1993). A inter-ação pela linguagem. Sao Paulo: Contexto.

(1997). O texto e a construção dos sentidos. Sao Paulo: Contexto. Lambrecht, K. (1994). Information Structure and Sentence Form. Topic, Focus, and the Mental Representations of Discourse Referents. Cambridge: Cambridge University Press.

LAMBRECHT K. (1994). Information Structure and Sentence Form. Topic, Focus, and the Mental Representations of Discourse Referents. Cambridge: Cambridge University Press. . (1986). Topic, Focus, and the Grammar of Spoken French, PhD Berkeley, Ann Arbor: UMI.

LANGACKER, R.W. (1990). Concept, Image and Symbol. The Cognitive Basis of Grammar, Berlin: De Gruyter.

LUFF, P. \& HEATH, C. (1993). System use and social organisation: observations on human-computer interaction in an architectural practice. In G. Button (Ed. ), Technology in working order: studies of work, interaction and technology. London: Routledge.

(2000). The collaborative production of computer commands in command and control. Int. J. Human-Computer Studies, 52, 669-699.

LUFF, P.; HEATH, C. \& JIROTKA, M. (2000). Surveying the scene: Technologies for everyday awareness and monitoring in control rooms. Interacting with Computers, 13, 193-228.

LYNCH, M. (1988). The externalized retina: Selection and mathematization in the visual documentation of objects in the life sciences. Human Studies, 11, 201-234. 
MONDADA, L. (1994). Verbalisation de l'espace et fabrication du savoir: Approche linguistique de la construction des objets de discours, Lausanne: Université de Lausanne, 670p.

(2001a). "Intervenir à distance dans une opération chirurgicale: l'organisation interactive d'espaces de participation". Bulletin Suisse de Linguistique Appliquée, 74, 33-56.

. (2001b). "Pour une linguistique interactionnelle", Marges Linguistiques, no 1, mai. (http://www.marges-linguistiques.com) (tr esp. "Por una lingüística interaccional", Discurso y Sociedad, 3/3, 2001, 61-90)

. (2002). "Gestion du topic et organisation de la conversation", Cadernos de Estudos Lingüísticos, Campinas, 41, 7-38.

. (à paraître a). "Deixis spatiale, gestes de pointage et formes de coordination de l'action", In: Barbéris, J. -M. , Manes-Gallo, M. C. (éds. ), Verbalisation de l'espace et cognition située: la description d'itinéraires piétons, Paris: Editions CNRS.

. (à paraître b). "Parler topical et organisation séquentielle: l'apport de l'analyse conversationnelle". Verbum.

(à paraître c). "Seeing for Saying. On the Discursive Construction of Knowledge in Travel Writing", in H. Schulz-Forberg (ed. ), Unravelling Civilisation. European Travel and Travel Writing.

(à paraître d). "Working with video: how surgeons produce video records of their actions", Visual Studies (special issue edited by M. Ball).

NELSON, R.J. (1992). Naming and Reference. The Link of Word to Object. London: Routledge.

OOSTEN VAN, J. (1986). The Nature of Subjects, Topics and Agents: A Cognitive Explanation, Bloomington: IULC.

PEKAREK, S. (1998). Linguistic forms and social interaction: Why do we specify referents more than is necessary for their identification? In J. Verschueren (Ed. ), Pragmatics in 1998. Selected Papers from the 6th International Pragmatics Conference (pp. 427-447). Antwerpen: IPrA.

SCHIFFRIN, D. (1987). Discourse Markers. Cambridge: Cambridge University Press.

SUCHMAN, L. (1996). Constituting shared workspaces. In D. Middleton \& Y. Engestrom (Eds. ), Cognition and Communication at Work. Cambridge: Cambridge University Press. 